\title{
Manual of \\ Cardiac Surgery
}




\section{Bradley J. Harlan, MD}

Chief, Section of Thoracic and Cardiovascular Surgery, Sutter Memorial Hospital, Sacramento, California; Assistant Clinical Professor of Surgery,University of California Davis Medical School, Sacramento, California; Formerly: Associate Professor of Surgery, Oregon Health Sciences University, Portland, Oregon

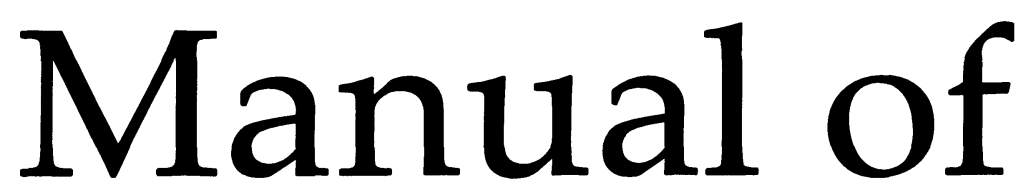

Springer-Verlag

New York Berlin Heidelberg London Paris

Tokyo Hong Kong Barcelona Budapest 


\section{Albert Starr, MD}

Professor of Surgery, Oregon Health Sciences University, Portland Oregon;

Director, Heart Institute at St. Vincent Hospital, Portland, Oregon

\section{Fredric M. Harwin, BFA, MS}

Harwin Studios, Portland, Oregon

\section{Cardiac Surgery Second Edition}

\section{With 363 Color Illustrations}

With Contributions by Alain Carpentier, MD

Professor of Cardiac Surgery, Hôpital Broussais, University of Paris, Paris, France 
Bradley J. Harlan, MD

5301 F Street, Suite 312

Sacramento, California 95819

USA
Albert Starr, MD

Starr-Wood Cardiac Group, P.C. 9155 SW Barnes Rd., Suite 240

Portland, Oregon 97225-6629

USA

\author{
Fredric M. Harwin \\ Harwin Studios \\ 9101 SW 15th Avenue \\ Portland, OR 97219 \\ USA
}

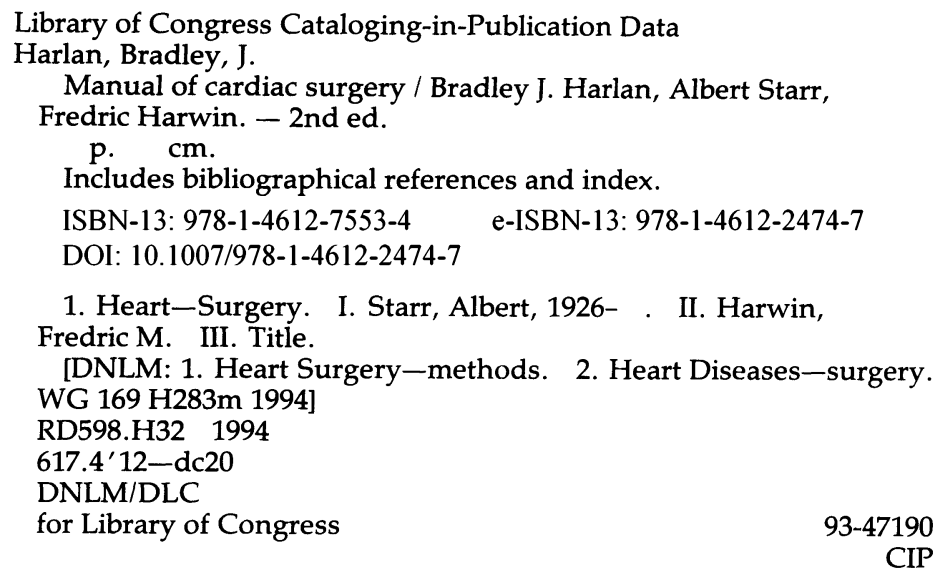

Printed on acid-free paper.

(C) 1995 by Springer-Verlag New York, Inc.

Softcover reprint of the hardcover 2nd edition 1995

All rights reserved. This work may not be translated or copied in whole or in part without the written permission of the publisher (Springer-Verlag New York, Inc., 175 Fifth Avenue, New York, NY 10010, USA), except for brief excerpts in connection with reviews or scholarly analysis. Use in connection with any form of information storage and retrieval, electronic adaptation, computer software, or by similar or dissimilar methodology now known or hereafter developed is forbidden.

The use of general descriptive names, trade names, trademarks, etc., in this publication, even if the former are not especially identified, is not to be taken as a sign that such names, as understood by the Trade Marks and Merchandise Marks Act, may accordingly be used freely by anyone.

While the advice and information in this book are believed to be true and accurate at the date of going to press, neither the authors nor the editors nor the publisher can accept any legal responsibility for any errors or omissions that may be made. The publisher makes no warranty, express or implied, with respect to the material contained herein.

Production managed by Jim Harbison and Natalie Johnson;

manufacturing supervised by Jacqui Ashri.

Typeset by Bytheway Typesetting Services, Norwich, NY. 
To our wives, Sharon, Victoria, and Sara and to our familes, whose love and support made this book possible 


\section{Preface}

The second edition of the Manual of Cardiac Surgery has the same goal as the first edition: to be detailed as possible regarding the process of cardiac surgery, from coverage of basic surgical technique to careful description of every essential step of each operation. Carefully conceived and superbly executed full-color illustrations are the primary vehicle for conveying this information.

The techniques chosen are those developed over three decades of clinical practice and resident training at the Oregon Health Sciences University and St. Vincent Hospital, Portland, Oregon, and Sutter Memorial Hospital, Sacramento, California. These techniques have served us well.

The first author moved from the Oregon Health Sciences University to Sutter Memorial Hospital in Sacramento in 1981. During the ensuing decade some of the techniques used by the first two authors have evolved in different directions. Where this is the case, the text indicates where the technique shown is used at the present time.

The text authors are proud to have the illustrator, Mr. Fredric Harwin, as coauthor. Mr. Harwin has been a significant contributor to the basic concepts and intellectual substance of the book. His art is a powerful instrument of education.

It is hoped that this second edition will have broad appeal to all persons caring for cardiac surgical patients. If it increases knowledge and understanding of cardiac surgery and thereby improves patient care, it will have met its main objective.

Bradley J. Harlan, MD

Albert Starr, MD

A medical illustrator must understand the philosophies and techniques of the scientist as well as those of the artist. For the second edition of Manual of Cardiac Surgery I worked with Dr. Harlan and Dr. Starr to create illustrations that allow the reader to visualize the surgical field as the surgeon sees it. For each illustration, I drew upon direct observation, operative photographs, fresh specimens, and, most importantly, extensive consultations with my surgeon co-authors. The sequence of creation was, first, discussion of desired illustrations, then a rough sketch, consultation with the surgeons, finished pencil drawing, another consultation, then finally the color rendering. These color renderings were then checked against actual surgery for accuracy in representation of tissues, instrumentation, tissue responses to manipulation, and consistency of representation. 
Close communication between artist and surgeon is essential to produce illustrations that are both anatomically and surgically correct and artistically viable. Such communication was present during the preparation of this book to convey not only the fundamentals of the operations, but also their most subtle details.

Fredric M. Harwin 


\section{Acknowledgments}

We wish to thank our colleagues who directly and indirectly inspired many concepts of this book and provided support and stimulation during its creation: from Sacramento, Edward A. Smeloff, George E. Miller Jr, Paul B. Kelly, Jr, Forrest L. Junod, Kenneth A. Ross, Kuppe G. Shankar, Douglas R. Schuch, Michael T. Ingram, and Eric A. Peper; and from Portland, James A. Wood, Richard D. Chapman, Aftab Ahmad, H. Storm Floten, Jeffrey Swanson, Hugh L. Gately, Duane S. Bietz, Hagop Hovaguimian, and Anthony P. Furnary.

Valuable review and modification of the manuscript was provided by $\mathrm{A}$. Glen Brooksby, Charles F. Collins, Jeffrey Leon, and Cornelius G. Wesseling for the chapter, "Anesthesia for Cardiac Surgery," and by Pat Brady and Gregory Meiling for the chapter "Conduct of Cardiopulmonary Bypass." We would like to thank Rodd Ambroson and Jeanne Koelling for their assistance with the production of illustrations. The creation of the illustrations was enhanced by the excellent photographs of our operations taken by Norman Meder. Gratitude is extended to Dee Sanchez, who helped greatly with the logistics required when a book is written and illustrated by authors living in different cities. We wish to thank Dr. Richard H. Egdahl for initiating the first edition of this project.

Finally, our thanks to Springer-Verlag, their superb staff, and our editor, William Day. We consider ourselves very fortunate to be beneficiaries of Springer-Verlag's longstanding expertise in the field of color reproduction and their strong commitment to quality. 


\section{Contents}

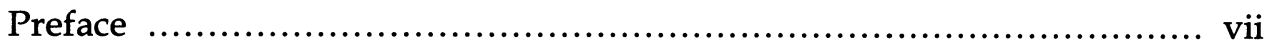

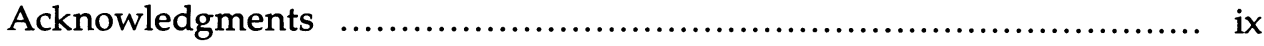

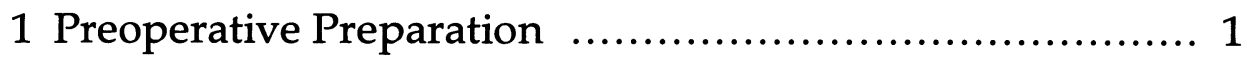

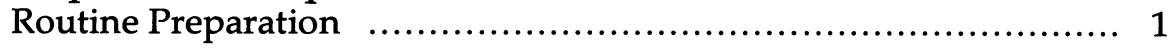

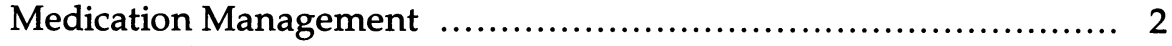

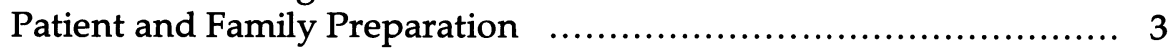

References ........................................................... 4

2 Anesthesia for Cardiac Surgery $\ldots \ldots \ldots \ldots \ldots \ldots \ldots \ldots \ldots \ldots$

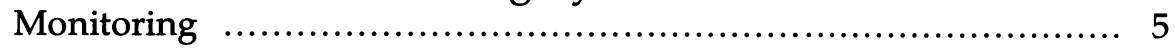

Premedication ....................................................... 6

Myocardial Protection: The Anesthesiologist's Role ................. 6

Anesthetic Agents .................................................. 8

Paralyzing Agents ............................................... 9

Vasodilators ......................................................... 9

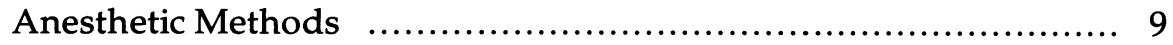

References ....................................................... 10

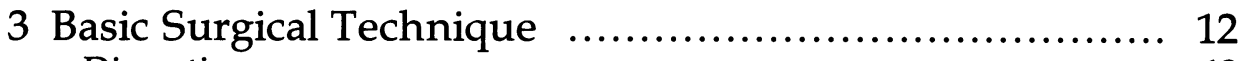

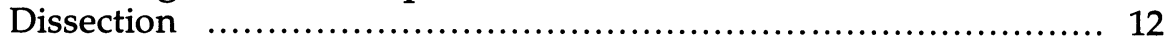

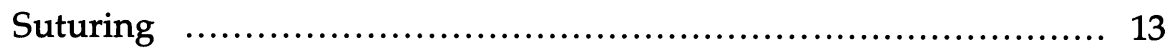

Additional Factors Affecting Technical Performance ............... 21

4 Preparation for Cardiopulmonary Bypass .............. 23

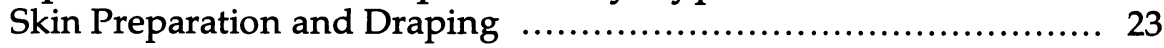

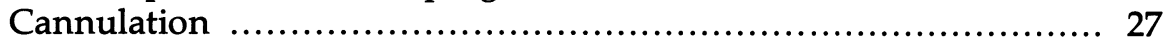

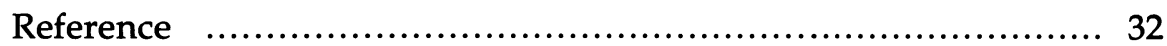

5 Conduct of Cardiopulmonary Bypass $\ldots \ldots \ldots \ldots \ldots \ldots . \ldots . \ldots 33$

Standard Bypass .................................................... 33

Cardiopulmonary Bypass in Neonates and Children ................ 37

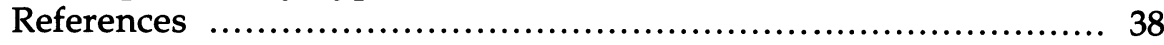

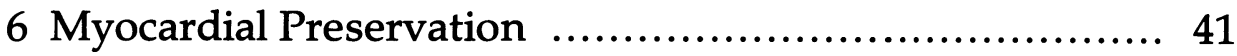

Operative (Mechanical) Coronary Perfusion $\ldots \ldots \ldots \ldots \ldots \ldots \ldots \ldots \ldots . \ldots . \ldots \ldots$

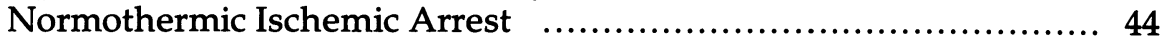

Ischemia Modified by Topical Hypothermia ......................... 48

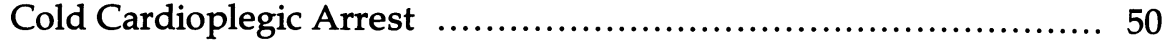




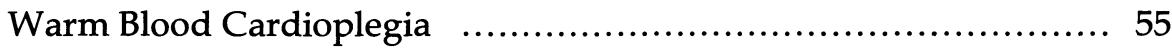

References .......................................................... 57

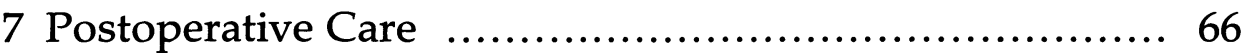

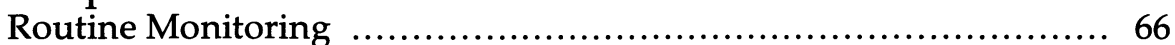

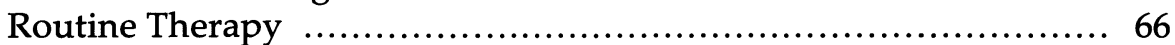

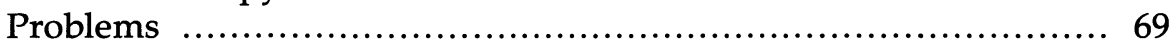

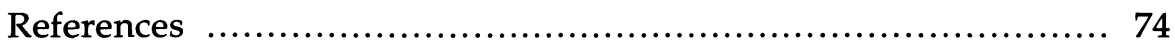

8 Low Cardiac Output: Pathophysiology

and Treatment .............................................. 77

Determinants of Cardiac Output ................................ 77

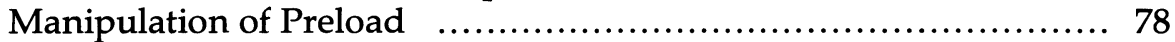

Manipulation of Afterload ......................................... 78

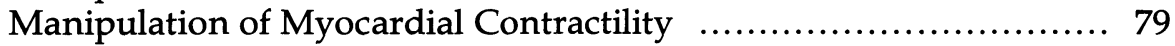

Indications for Intraaortic Balloon Counterpulsation $\ldots \ldots \ldots \ldots \ldots \ldots . . .60$

Technique of Intraaortic Balloon Insertion $\ldots \ldots \ldots \ldots \ldots \ldots \ldots \ldots \ldots \ldots . \ldots 10$

References ........................................................ 81

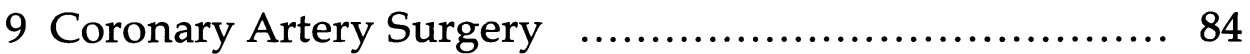

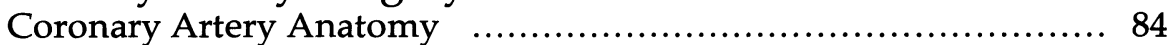

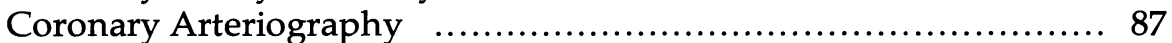

Noninvasive Methods of Diagnosis of Coronary Artery Disease ... 92

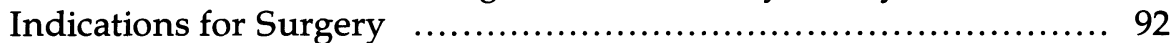

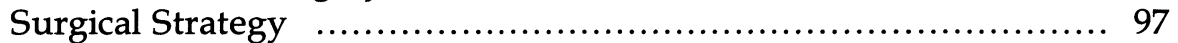

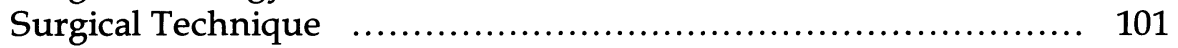

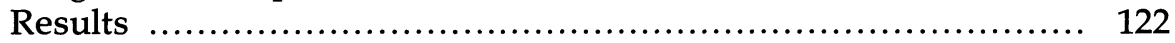

References ................................................. 124

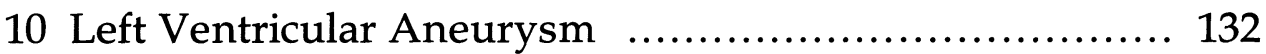

Resection ........................................................ 132

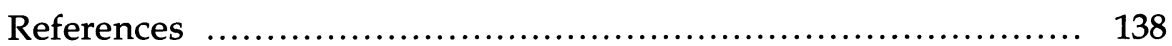

11 Postinfarction Ventricular Septal Defect ............... 142

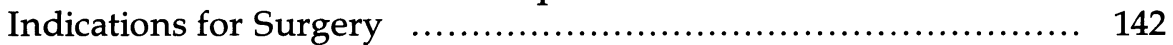

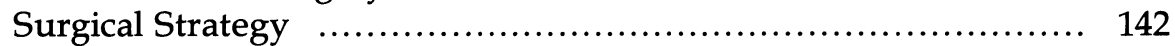

Surgical Anatomy ............................................... 143

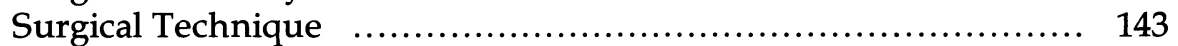

Results .......................................................... 145

References ...................................................... 146

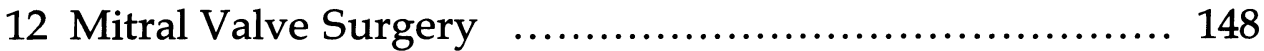

With Alain Carpentier

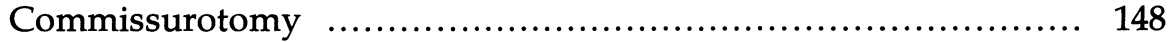

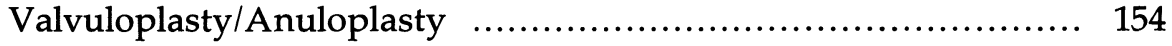

Replacement ..................................................... 163

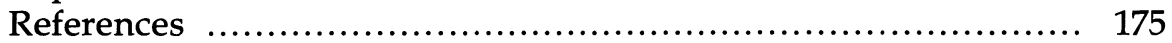

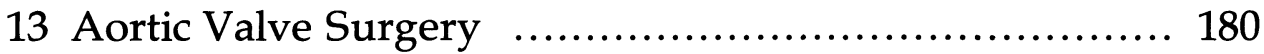

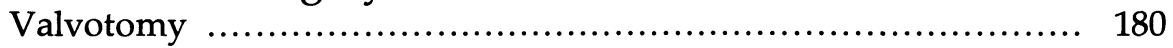

Replacement ................................................... 182

Surgical Relief of Other Forms of Left Ventricular

Outflow Obstruction ............................................. 201

References ......................................................... 202 


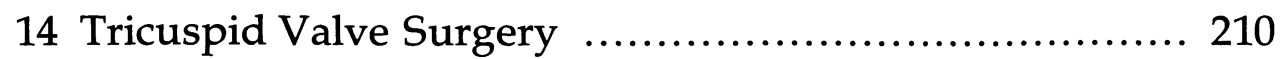
With Alain Carpentier

Anuloplasty ..................................................... 210

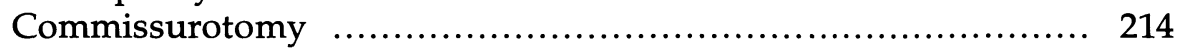

Replacement .................................................... 215

References .................................................. 216

15 Patent Ductus Arteriosus ................................. 218

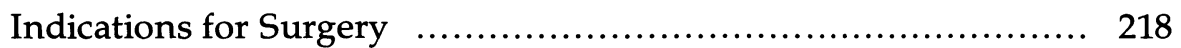

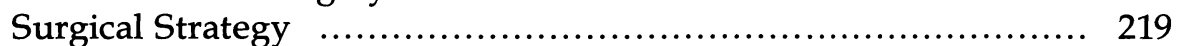

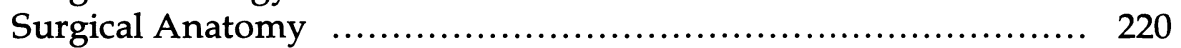

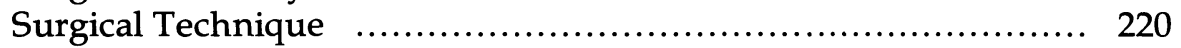

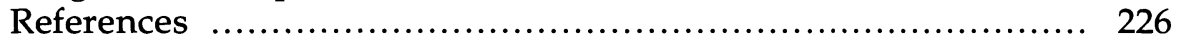

16 Coarctation of the Aorta .................................. 228

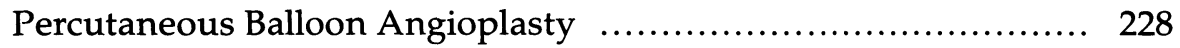

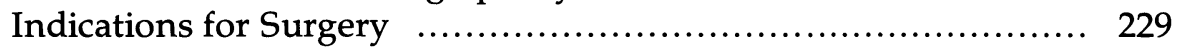

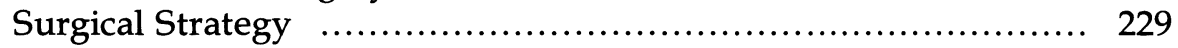

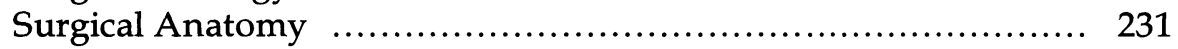

Surgical Technique .......................................... 233

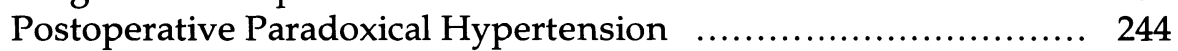

Results .......................................................... 244

References ....................................................... 245

17 Systemic-Pulmonary Shunts ........................... 249

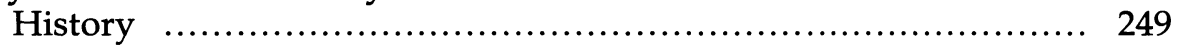

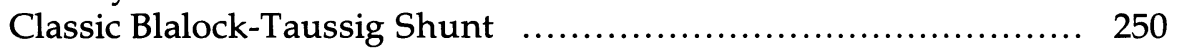

Modified Blalock-Taussig Shunt ................................ 254

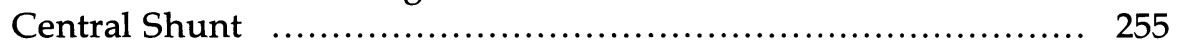

References ..................................................... 258

18 Pulmonary Valve Stenosis ............................... 260

Percutaneous Balloon Valvuloplasty $\ldots . \ldots \ldots \ldots \ldots \ldots \ldots \ldots \ldots \ldots . \ldots . \ldots 260$

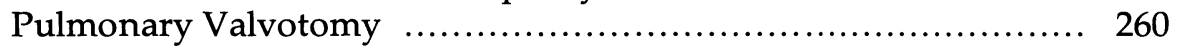

References ..................................................... 264

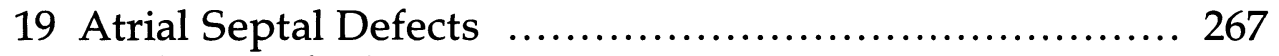

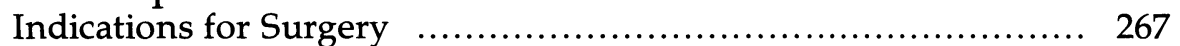

Sinus Venosus Defect .......................................... 268

Results ............................................................. 269

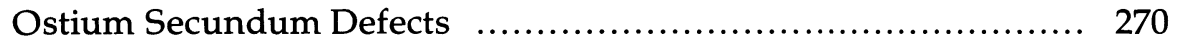

Ostium Primum Defect ......................................... 272

References ................................................. 277

20 Complete Atrioventricular Canal $\ldots \ldots \ldots \ldots \ldots \ldots \ldots \ldots \ldots . \ldots . \ldots . \ldots 280$

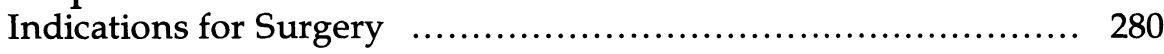

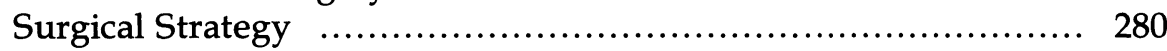

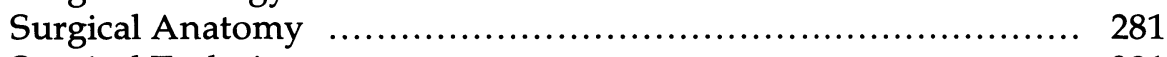

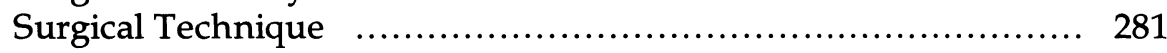

Results .......................................................... 285

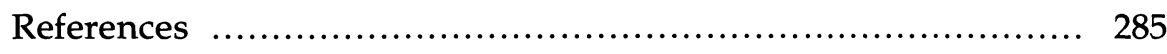

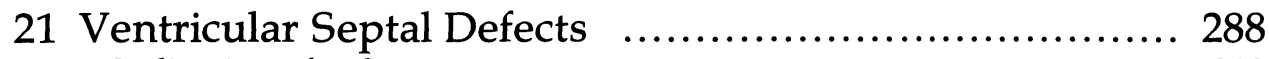

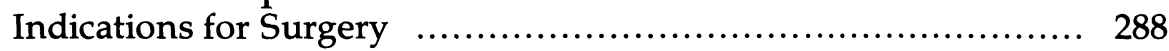


Choice of Operation . ............................................... 289

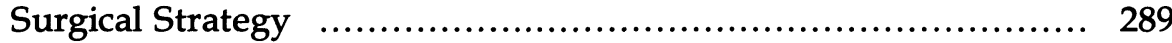

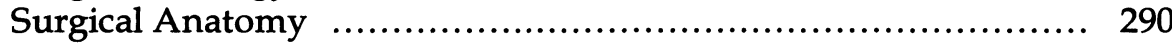

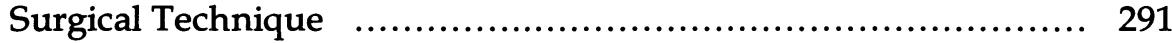

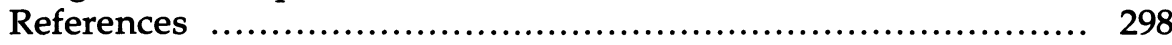

22 Tetralogy of Fallot ..................................... 301

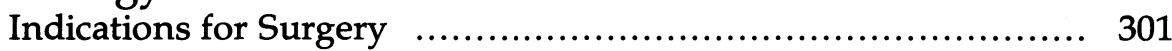

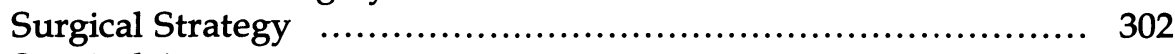

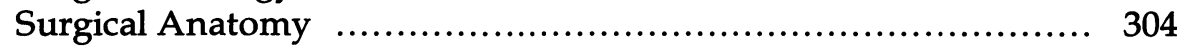

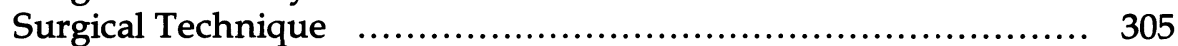

Late Results ...................................................... 311

References ........................................................ 311

23 Transposition of the Great Arteries $\ldots \ldots \ldots \ldots \ldots \ldots . \ldots . \ldots . \ldots 15$

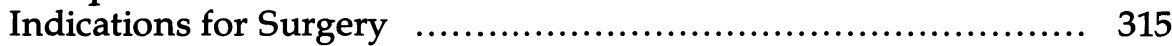

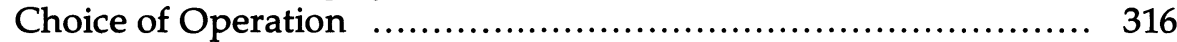

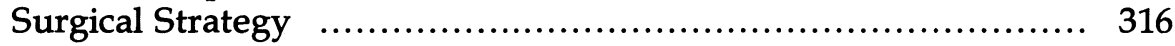

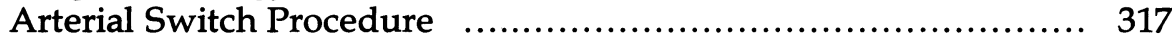

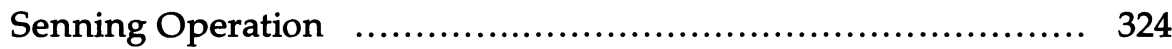

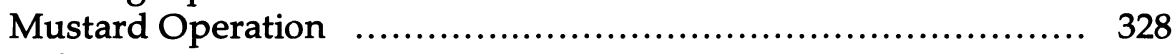

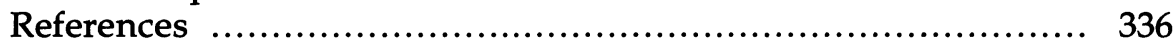

24 Total Anomalous Pulmonary Venous Connection ....... 342

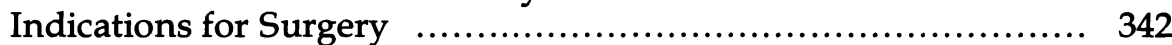

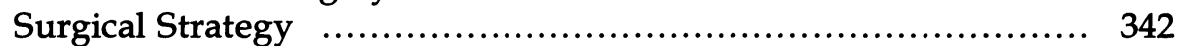

Supracardiac Type ............................................. 342

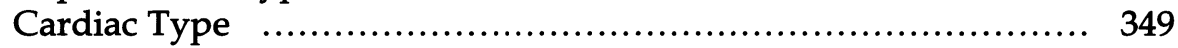

Infracardiac Type ........................................... 351

Results ......................................................... 353

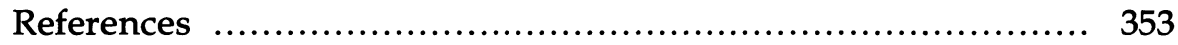

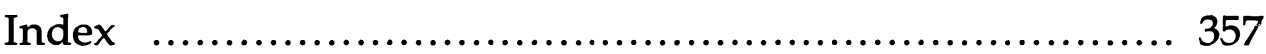

